\title{
Compressive Sparsity Order Estimation for Wideband Cognitive Radio Receiver
}

\author{
Shree Krishna Sharma, Student Member, IEEE, Symeon Chatzinotas, Senior \\ Member, IEEE and Björn Ottersten, Fellow, IEEE
}

\begin{abstract}
Compressive Sensing (CS) has been widely investigated in the Cognitive Radio (CR) literature in order to reduce the hardware cost of sensing wideband signals assuming prior knowledge of the sparsity pattern. However, the sparsity order of the channel occupancy is time-varying and the sampling rate of the CS receiver needs to be adjusted based on its value in order to fully exploit the potential of CS-based techniques. In this context, investigating blind Sparsity Order Estimation ( $\mathrm{SOE}$ ) techniques is an open research issue. To address this, we study an eigenvalue-based compressive SOE technique using asymptotic Random Matrix Theory. We carry out detailed theoretical analysis for the signal plus noise case to derive the asymptotic eigenvalue probability distribution function (aepdf) of the measured signal's covariance matrix for sparse signals. Subsequently, based on the derived aepdf expressions, we propose a technique to estimate the sparsity order of the wideband spectrum with compressive measurements using the maximum eigenvalue of the measured signal's covariance matrix. The performance of the proposed technique is evaluated in terms of normalized SOE Error (SOEE). It is shown that the sparsity order of the wideband spectrum can be reliably estimated using the proposed technique for a variety of scenarios.
\end{abstract}

Index Terms: Sparsity Order Estimation, Random Matrix Theory, Wideband Cognitive Radio, Compressive Sensing

Copyright (c) 2014 IEEE. Personal use of this material is permitted. However, permission to use this material for any other purposes must be obtained from the IEEE by sending a request to pubs-permissions@iee.org.

This work was supported by the National Research Fund, Luxembourg under AFR (Aids Training-Research) grant for a PhD project (Reference 3069102) and the CORE project "CO2SAT". The partial results of this paper were presented in International Conference on Communication, Sydney, 10-14 June, 2014.

The authors are with the Interdisciplinary Centre for Security, Reliability and Trust (SnT) (http://www.securityandtrust.lu), University of Luxembourg, L-2721, Luxembourg. Email: \{shree.sharma, symeon.chatzinotas, bjorn.ottersten\}@uni.lu. 


\section{INTRODUCTION}

Cognitive Radio (CR) communications is considered a promising solution in order to enhance the spectral efficiency of future wireless systems [1]. Among several CR techniques proposed in the literature, Spectrum Sensing (SS) is an important mechanism in order to acquire the spectrum awareness required by the CRs. Existing SS techniques mostly focus on the detection of narrowband signals considering a single radio channel [2-5]. However, in practical scenarios, the CRs need to detect and acquire information about a wide spectrum band in order to utilize the spectrum efficiently. Further, CRs do not have prior knowledge about the PU's signal and channel. In this aspect, investigating efficient blind spectrum awareness techniques is an important and relevant research challenge.

The key challenge of SS is the detection of weak wideband signals hidden in thermal noise with a sufficiently large probability of detection. The sensing Radio Frequency (RF) chain of a CR receiver should be able to receive a wideband signal, sample it using a high speed Analog to Digital Converter (ADC) and perform measurements for the detection of PU signals. The wideband RF signal received by the antenna of an RF front-end includes signals from adjacent and spatially separated transmitters, and from transmitters operating at a wide range of power levels and channel bandwidths. In this context, one of the main challenges in implementing a wideband CR is the design of the RF front-end [6,7]. Further, the main limitation in an RF front-end's ability to detect weak signals is its Dynamic Range (DR). For this purpose, the wideband sensing requires multi-GHz speed ADCs, which together with high resolution (of 12 or more bits) might be infeasible with current technology $[8,9]$.

In the above context, Compressive Sensing (CS) has emerged as an important technique which can significantly reduce the acquisition cost at the CR node [10,11]. According to CS theory, certain signals can be recovered from far fewer samples or measurements than those required by conventional methods [12-14]. Most of the CS literature has focused on improving the speed and accuracy of recovering the original sparse signal from compressive measurements [15-17]. In the context of CR networks, CS techniques are suitable for acquiring the spectrum usage information in a wide spectrum band as in many cases the spectrum occupancy is sparse in the frequency and time domains [18].

\section{A. Motivation and Contributions}

In most of the existing CR-related CS contributions, the original sparse signal is reconstructed generating Nyquist rate samples from the compressed samples and subsequently, SS is carried out [10,19]. However, 
the reconstruction process in a CS-based CR requires significant computational complexity [20] and from the SS point of view, it's not necessary to reconstruct the entire sparse signal. In this direction, CS has been applied in [21] and [22] for signal detection purpose using compressed measurements rather than reconstructing the original signal. In contrast to [21] and [22], we are interested in estimating the sparsity order of the wideband spectrum which can be subsequently used for implementing adaptive CS-based CR receivers.

Furthermore, in most of the CS-related contributions, it is assumed that the wideband signal is sparse in some domain and the number of measurements is kept fixed based on the assumed sparsity order. However, in compressive wideband systems, the required number of measurements to achieve a successful recovery rate proportionally varies with the sparsity order of wideband signals $[11,23]$. In this context, Sparsity Order Estimation (SOE) is crucial in order to choose the appropriate number of measurements. In addition, in most of the contributions, it is assumed that the sparsity order of the signal is known beforehand. However, in the context of CR networks, this prior information is not available at the CR sensor and has to be estimated. In the above context, a two-step CS algorithm has been proposed recently in [23], in which the sparsity order is estimated in the first step and the total number of collected samples is adjusted in the second step based on the estimated sparsity order. However, the considered estimation approach is based on Monte Carlo simulations and no analytical method has been presented for estimating the sparsity order of the wideband signals. Further, the proposed experimental method for SOE in [23] requires the reconstruction of the original sparse signal.

To address the above issues, we provide the following contributions in this paper.

1) We propose a Multiple Measurement Vector (MMV) model to study the SOE problem. To estimate the sparsity order, we consider an eigenvalue-based approach using the eigenvalues of the CS measurement vector since it requires no prior information about the PU signals neither the knowledge of channel nor the noise covariance [24]. Our theoretical analysis differs from [25] since our analysis is based on the MMV model instead of the Single Measurement Vector (SMV) model considered in $[25]$.

2) We derive theoretical expressions for asymptotic eigenvalue probability distribution function (aepdf) of the measured signal's covariance matrix for three different scenarios using asymptotic Random Matrix Theory (RMT). In the first case, we consider a simple case of constant received power across 
all active carriers in the considered wideband spectrum. However, in practice, the transmitted power is different across multiple carriers due to spatial separation of PUs and frequency selective channels. To address this scenario, we also study the case where the received power levels across the carriers are different but follow a specific distribution. Further, the non-zero entries across the multiple measurement vectors may be correlated in practice due to channel correlation or imperfections in frequency selective filters present at the CR node. To address this specific issue, we consider the correlated MMV scenario where the non-zero entries across multiple measurement vectors are correlated.

3) Based on the derived aepdf expressions, we propose a technique in order to estimate the sparsity order of the channel occupancy within the considered wideband spectrum. In this context, we consider multiple subbands within the considered wideband spectrum and represent each subband with a center carrier frequency.

4) We study a tradeoff between estimation performance (expressed in terms of SOE Error (SOEE)) and the hardware cost (number of measurements).

\section{B. Related Work}

1) CS-based Signal Acquisition: Various sub-Nyquist wideband sensing techniques have been proposed in the literature assuming sparse spectrum occupancy over a wideband spectrum [10,26-28]. In [10], CS theory has been applied to the CRs for acquiring wideband signals using sub-Nyquist sampling rates considering the sparseness of the signal spectrum in open-access network. The contribution in [26] proposes several methods for low-rate sampling of continuous-time sparse signals in shift-invariant spaces. A multirate sub-Nyquist sampling system has been proposed in [27] to perform wideband SS considering several sub-Nyquist sampling branches in each sensing node. The authors in [28] study the blind sub-Nyquist sampling of multiband signals, whose unknown frequency support occupies only a small portion of a wide spectrum. In [29], a sequential CS approach has been proposed where observations are available in a sequence and can be stopped as soon as there is reasonable certainty of correct reconstruction.

2) Signal Recovery Algorithms and Hardware Architectures: The basic CS theory starts with the SMV problem and is extensively applied in magnetic resonance imaging, underwater acoustic channel estimation and direction of arrival (DoA) estimation [30,31]. Several algorithms have been developed for solving the SMV problem including Orthogonal Matching Pursuit (OMP) [15] and its variants, sparse reconstruction 
by separable approximation (SpaRSA) algorithm [32], $l_{1}$ regularized least squares $\left(l_{1}\right.$-LS) [33], focal undetermined system solve (FOCUSS) class of algorithms [34], and least square absolute shrinkage and selection operator (LASSO) algorithm [35]. In comparison to the SMV case, the support recovery rate can be significantly enhanced using the MMV method [37]. Several MMV algorithms have been proposed in the literature including M-OMP [36], M-FOCUSS, $l_{1} / l_{2}$ minimization [37], and Multiple Sparse Baysian Learning (M-SBL) [38]. Further, several hardware architectures have been proposed and implemented in the literature enabling the acquisition of compressive samples in practical settings $[28,39,40]$.

3) Support Recovery Techniques: Several contributions focus on the support recovery problem instead of recovering the original sparse signal [25,41-45]. The contributions [43] and [44] focus on necessary and sufficient conditions for exact recovery of the sparsity pattern. In [42], the support recovery problem is studied in the context of the MMV model and it is shown that the structure of the matrix formed by the nonzero entries has a significant role in the performance limits of the support recovery. The contribution in [41] investigates a tradeoff between sampling rate and the detection error focusing on the high-dimensional setting. In addition, [45] provides sufficient conditions for stable recovery assuming the prior knowledge of the partial support information. Moreover, the recent contribution in [25] studies a sparse support recovery problem using an asymptotic RMT approach.

\section{Structure and Notation}

This paper is structured as follows: Section II describes the signal model and describes three different study cases. Section III presents theoretical analysis for constant power case using RMT. Similarly, Section IV provides theoretical analysis for varying power case and Section V includes the analysis for correlated MMV case. Section VI presents the proposed eigenvalue-based SOE technique based on the derived aepdf expressions. Section VII evaluates the performance of the proposed SOE technique for the considered scenarios with numerical simulations. Section VIII presents conclusions and proposes future work in this domain. The appendix includes some preliminaries on random matrix transforms and proofs of the theorems.

Throughout this paper, boldface upper and lower case letters are used to denote matrices and vectors respectively, $\mathbb{E}_{\mathbf{X}}[\cdot]$ denotes expectation over $\mathbf{X}, \mathbb{C}$ denotes complex numbers, $(\cdot)^{T}$ and $(\cdot)^{\dagger}$ denote the transpose and the conjugate transpose respectively, $(\cdot)^{*}$ represents the complex conjugate, $(z)^{+}$denotes $\max (0, z), \mathcal{P}[\cdot]$ denotes the probability, $\mathbf{X}_{i, j}$ denotes the $(i, j)$ th element of $\mathbf{X}, f_{\mathbf{X}}(\cdot)$ denotes the eigenvalue 
distribution function of $\mathbf{X}, \mathbf{R}_{\mathbf{X}}$ represents the covariance matrix of $\mathbf{X}, \hat{\mathbf{R}}_{\mathbf{X}}$ represents the sample covariance of $\mathbf{X}, \mathcal{S}_{\mathbf{X}}$ represents the Stieltjes transform of $\mathbf{X}, \mathcal{R}_{\mathbf{X}}$ represents the $\mathrm{R}$ transform ${ }^{1}, \Sigma_{\mathbf{X}}$ represents the $\Sigma$ transform and $\eta_{\mathbf{X}}$ represents the $\eta$ transform [46].

\section{SyStem AND SignAl Model}

Let us consider a total bandwidth of $W \mathrm{~Hz}$ with $N$ number of carriers each having $W / N$ channel bandwidth. Since all the carriers may not be occupied all the time [18], we assume sparse channel occupancy in the considered wideband spectrum. We define sparsity order, let us denote by $\sigma$, as the ratio of the number of the occupied carriers to the total number of carriers over the considered wideband spectrum. Further, we define another parameter $\rho$ as the compression ratio, which depends on the number of measurements as discussed later. The signal model used in this paper has been inspired from the model used in [25] which focuses on the input-output mutual information and the support recovery rate in the asymptotic limit. For our analysis in this paper, we extend the SMV model of [25] into the MMV model as described below.

Let us consider the following complex valued observation model for the SMV problem as considered in [25]

$$
\mathbf{y}=\mathbf{A U X b}+\mathbf{z}
$$

where $\mathbf{A}$ is an $N \times N$ diagonal matrix with independent and identically distributed (i.i.d.) diagonal Bernoulli distributed elements i.e., $\mathcal{P}\left[\mathbf{A}_{i, i}=1\right]=\rho=1-\mathcal{P}\left[\mathbf{A}_{i, i}=0\right]$, where $\rho=\frac{1}{N} \mathbb{E}_{\mathbf{A}}[M]=\frac{1}{N} \bar{M}$ denotes the average fraction of non-zero diagonal elements of $\mathbf{A}$ to the total number of diagonal elements and is equivalent to the ratio of the dimensions of the measurement matrix considered in [20], $\mathbf{U}$ is an $N \times N$ random matrix having i.i.d. elements with zero mean and variance $1 / N$, and an $N \times N$ combined matrix AU denotes the compressed sensing matrix. Further, $\mathbf{X}=\operatorname{diag}(\mathbf{x})$, with $\mathbf{x}$ being an $1 \times N$ complex Gaussian vector having i.i.d. elements with zero mean and variance $1 / N, \mathbf{b}$ is an $N \times 1$ vector with i.i.d. complex components $b_{i}$ distributed with Bernoulli distribution i.e., $\mathcal{P}\left[b_{i}=1\right]=\sigma=1-\mathcal{P}\left[b_{i}=0\right]$ with its non-zero elements defining the support of Bernoulli-Gaussian vector $\mathbf{X b}$, whose sparsity (average fraction of non-zero elements) is equal to $\sigma=\frac{1}{N} \mathbb{E}_{\mathbf{b}}[M]$, and $\mathbf{z}$ is an i.i.d. complex Gaussian $N \times 1$ vector with components $z_{i} \sim \mathcal{C N}(0,1)$. It should be noted that $\mathbf{X b}$ denotes the sparse vector considered for

\footnotetext{
${ }^{1}$ Readers should not confuse the $\mathrm{R}$ transform notation $\mathcal{R}$ with the covariance matrix notation $\mathbf{R}$.
} 
the standard CS formulation in the literature [20]. In model (1), the non-zero diagonal elements of A define the components of the product $\mathbf{U X b}$ for which a noisy measurement is acquired and the parameter $\rho$ indicates the sampling rate i.e., average fraction of the observed components. Further, as in [25], we consider the covariance $\mathbf{U}^{\dagger} \mathbf{A}^{\dagger} \mathbf{A} \mathbf{U}$ to be free from any deterministic Hermitian matrix [46].

In contrast to the above SMV problem, we are interested in analyzing the MMV scenario, where the combination of concatenated multiple measurement vectors has been represented in the form of a matrix Y. We consider $N \times N$ sensing matrix AU consisting of $\bar{M}=\rho N$ number of non-zero rows. This is equivalent to the scenario with a $\mathrm{CR}$ node equipped with $\bar{M}$ number of frequency selective filters considered in [47], where $\bar{M}$ filters are used to measure $\bar{M}$ different linear combinations of the received signals of all $N$ carriers. In practice, the frequency selective surfaces can be used to realize frequency filtering by designing a planar periodic structure with a unit element size around half wavelength of the frequency of interest. Further, the bandwidth of a subband can be dealt by finding a suitable shape of the unit elements through the initial test procedure [47]. Let $N$ also be the number of samples collected by a sensor during the measurement process and therefore each measurement vector of $\mathbf{Y}$ contains $N$ number of samples. We assume that the channel occupancy status remains unchanged during the period of measurement. In this context, we extend the SMV problem (1) into the following MMV model

$$
\mathbf{Y}=\mathbf{A} \mathbf{U} \mathbf{P}^{1 / 2} \boldsymbol{\Theta}^{1 / 2} \mathbf{B X}+\mathbf{Z}=\mathbf{A} \mathbf{U P}^{1 / 2} \boldsymbol{\Theta}^{1 / 2} \mathbf{S}+\mathbf{Z}
$$

where $\mathbf{B}=\operatorname{diag}(\mathbf{b})$ is an $N \times N$ diagonal matrix with the diagonal having i.i.d. Bernoulli distributed elements i.e., $\mathcal{P}\left[\mathbf{B}_{i, i}=1\right]=\sigma=1-\mathcal{P}\left[\mathbf{B}_{i, i}=0\right], \mathbf{X}$ is an $N \times N$ random matrix having i.i.d. entries with zero mean and variance $1 / N$. The definitions of matrices $\mathbf{A}$ and $\mathbf{U}$ are same as the ones defined earlier in the SMV model. Further, $\mathbf{P}$ is an $N \times N$ diagonal matrix and its diagonal entries correspond to the varying power levels received across different carriers within the considered spectrum, and $\Theta^{1 / 2}$ is the square root of the correlation matrix $\Theta$ and its elements are drawn from an exponential correlation model as defined later in Section $\mathrm{V}$. The $N \times N$ matrix $\mathbf{S}=\mathbf{B X}$ is a sparse signal matrix with uniform sparsity (sparsity order $\sigma$ ) across all the columns. It can be noted that $\mathbf{Y}$ contains $\bar{M}=\rho N$ number of non-zero rows and each non-zero row contains $N$ number of samples. We assume that the matrices $\mathbf{A}, \mathbf{U}, \mathbf{B}, \mathbf{X}$ and $\mathrm{Z}$ are mutually independent. The sensing matrix $\mathrm{AU}$ is assumed to be known by the receiver.

Assuming that the source signal is independent from the noise, the covariance matrix of the measured 
signal, denoted by $\mathbf{R}_{\mathbf{Y}}$, can be calculated as [3]:

$$
\mathbf{R}_{\mathbf{Y}}=\mathbb{E}\left[\mathbf{Y} \mathbf{Y}^{\dagger}\right]=\mathbb{E}\left[\left(\mathbf{A U} \mathbf{P}^{1 / 2} \mathbf{\Theta}^{1 / 2} \mathbf{B X}\right)\left(\mathbf{A} \mathbf{U} \mathbf{P}^{1 / 2} \mathbf{\Theta}^{1 / 2} \mathbf{B X}\right)^{\dagger}\right]+\mathbb{E}\left[\mathbf{Z} \mathbf{Z}^{\dagger}\right]
$$

In this paper, we are interested in finding out the eigenvalue distribution $f(\lambda)$ of $\mathbf{R}_{\mathbf{Y}}$. Since all the matrices $\mathbf{A}, \mathbf{U}, \mathbf{B}, \mathbf{X}$ and $\mathbf{Z}$ are square, $f_{\mathbf{R}_{\mathbf{Y}}}(\lambda)=f_{\overline{\mathbf{R}}_{\mathbf{Y}}}(\lambda)$, where $\overline{\mathbf{R}}_{\mathbf{Y}}=\mathbf{P \Theta E}\left[\mathbf{U}^{\dagger} \mathbf{A}^{\dagger} \mathbf{A} \mathbf{U B X} \mathbf{X}^{\dagger} \mathbf{B}^{\dagger}\right]+\mathbb{E}\left[\mathbf{Z Z} \mathbf{Z}^{\dagger}\right]=$ $\mathbf{P} \Theta \mathbf{R} R_{1}+\mathbf{R}_{\mathbf{Z}}$ with $\mathbf{R}=\mathbb{E}\left[\mathbf{U}^{\dagger} \mathbf{A}^{\dagger} \mathbf{A U}\right], \mathbf{R}_{1}=\mathbb{E}\left[\mathbf{B X X} \mathbf{B}^{\dagger}\right]$ and $\mathbf{R}_{\mathbf{Z}}=\mathbb{E}\left[\mathbf{Z} \mathbf{Z}^{\dagger}\right]$. In practice, the covariance matrix $\mathbf{R}_{\mathbf{Y}}$ is not available and we have to rely on the sample covariance matrix. Let us define the sample covariance matrices of the measured signal and noise as: $\hat{\mathbf{R}}_{\mathbf{Y}}(N)=\frac{1}{N} \mathbf{Y} \mathbf{Y}^{\dagger}$ and $\hat{\mathbf{R}}_{\mathbf{Z}}(N)=\frac{1}{N} \mathbf{Z} \mathbf{Z}^{\dagger}$. Similarly, let $\hat{\mathbf{R}}$ and $\hat{\mathbf{R}}_{1}$ be the sample covariance matrices corresponding to the covariance matrices $\mathbf{R}$ and $\mathbf{R}_{1}$ respectively. It can be noted that $\hat{\mathbf{R}}$ and $\hat{\mathbf{R}}_{1}$ are asymptotically free from any deterministic matrix for the considered $\mathbf{X}$ and $\mathbf{U}$ [25]. As mentioned in Section I, we are interested in studying the following three specific cases.

Case 1: Constant Power: We consider equal received power across all the carriers and uncorrelated non-zero entries across the multiple measurement vectors of $\mathbf{Y}$. In this case, the observation model (2) reduces to the following

$$
\mathbf{Y}=\mathbf{A} \mathbf{U} \sqrt{p} \mathbf{B X}+\mathbf{Z}
$$

where $p$ denotes the constant power across all the carriers. Since we assume normalized noise variance, $\mathrm{SNR} \equiv p$. The value of SNR is assumed to be known and it can be acquired through SNR estimation techniques like in $[48,49]$. The detailed analysis of this case is presented in Section III.

Case 2: Varying Power: The received power levels across all the carriers vary but are assumed to follow a known distribution. Further, we consider uncorrelated non-zero entries across the multiple measurement vectors of $\mathbf{Y}$. In this case, the observation model (2) reduces to the following

$$
\mathbf{Y}=\mathbf{A U P} \mathbf{P}^{1 / 2} \mathbf{B X}+\mathbf{Z}
$$

where $\mathbf{P}$ is an $N \times N$ diagonal matrix with its diagonal entries corresponding to the varying power levels received across different carriers as defined earlier. The detailed analysis of this case is presented in Section IV.

Case 3: Correlated MMV: We consider the correlated scenario in which the non-zero entries across the multiple measurement vectors are correlated and assume that the received power across each carrier is 
constant. In this case, the observation model (2) reduces to the following

$$
\mathbf{Y}=\mathbf{A} \mathbf{U} \Theta^{1 / 2} \sqrt{p} \mathbf{B X}+\mathbf{Z}
$$

where $\Theta^{1 / 2}$ is represents the square root of the correlation matrix $\Theta$ and the its elements are drawn from an exponential correlation model. The detailed analysis of this case is presented in Section V.

Remark 2.1: It can be noted that (4), (5), and (6) are special cases of (2) and the solutions of these cases can be derived from the solution of (2). However, it is complex to obtain the closed form expression of the Stieltjes transform of (2) due to the involvement of several matrices in the first term. Due to this reason, we solve three specific cases in this paper. Further, in the following sections, we have provided coefficients for the ploymonials of Stieltjes transforms of cases 1 and 3, while the closed form polymonial for the Stieltjes transform of case 2 involves several higher order coefficients and is not presented in this paper for the sake of conciseness.

\section{Analysis for Constant Power Case}

Assuming that signal and noise are uncorrelated with each other, for large values of $N$, the measured signal's sample covariance matrix can be written using the following asymptotic approximation [3]:

$$
\lim _{N \rightarrow \infty} \hat{\mathbf{R}}_{\mathbf{Y}}(N) \approx p \hat{\mathbf{R}} \hat{\mathbf{R}}_{1}+\hat{\mathbf{R}}_{\mathbf{Z}}
$$

For the considered constant received power case, the aepdf of the measured signal's sample covariance matrix given by (7) can be used to estimate the sparsity order over the considered wideband spectrum as illustrated later in Section VI. In this context, our aim is to find the aepdf of $\hat{\mathbf{R}}_{\mathbf{Y}}(N)$. However, due to noncommutative nature of random matrices (Example 2.33, [46]), it's not straightforward to calculate the eigenvalue distribution of $\hat{\mathbf{R}}_{\mathbf{Y}}(N)$ by knowing the individual eigenvalue distributions of $\hat{\mathbf{R}}, \hat{\mathbf{R}}_{1}$ and $\hat{\mathbf{R}}_{\mathbf{Z}}$. Using free probability analysis, the asymptotic spectrum of the sum or product can be obtained from the individual asymptotic spectra without involving the structure of the eigenvectors of the matrices (Section 2.4, [46]). According to free probability theory of random matrices, $\Sigma$ transform follows the multiplicative free convolution property [53] while the $\mathrm{R}$ transform follows the additive free convolution property [54] (For the definitions of $\mathrm{R}$ and $\Sigma$ transforms, please see Appendix A). In this context, the free convolution properties of $\Sigma$ and $\mathrm{R}$ transforms can be used to find out the eigenvalue distribution of $\hat{\mathbf{R}}_{\mathbf{Y}}(N)$ as illustrated later in the Appendix (See Appendixes B, C and D). By applying these properties in (7) and with the help of Theorems 28, 29 and 33 (in Appendix A), the Stieltjes transform of asymptotic 
density function of $\hat{\mathbf{R}}_{\mathbf{Y}}(N)$ can be obtained. Subsequently, the derived Stieltjes transform is used to find the aepdf of $\hat{\mathbf{R}}_{\mathbf{Y}}(N)$ using Theorem 8.9 (in Appendix A), which is then subsequently used to estimate the sparsity order in our considered problem.

From [46, Theorem 2.39], the $\eta$ transform of $\hat{\mathbf{R}}$ satisfies the following relation

$$
1=\frac{1-\eta_{\hat{\mathbf{R}}}(z)}{1-\eta_{\mathbf{F}}\left(z \eta_{\hat{\mathbf{R}}}(z)\right)}
$$

with $\mathbf{F}=\mathbf{A}^{\dagger} \mathbf{A}$. Since $\mathbf{A}$ is diagonal with Bernoulli i.i.d. diagonal elements, its $\eta$ transform can be written as [25]

$$
\eta_{\mathbf{F}}(z)=\eta_{\mathbf{A}}(z)=1-\rho+\frac{\rho}{1+z}
$$

Using (9) in (8), the $\eta$ transform of $\hat{\mathbf{R}}$ is given by the positive solution of the following polymonial

$$
z \eta_{\hat{\mathbf{R}}}^{2}(z)-((1-\rho) z-1) \eta_{\hat{\mathbf{R}}}(z)-1=0
$$

Using the similar procedure, the $\eta$ transform of $\hat{\mathbf{R}}_{1}$ is given by the positive solution of the following polymonial

$$
z \eta_{\hat{\mathbf{R}}_{1}}^{2}(z)-((1-\sigma) z-1) \eta_{\hat{\mathbf{R}}_{1}}(z)-1=0
$$

Equation (10) corresponds to the $\eta$ transform of the $\mathbf{H H}^{\dagger}$ with $N \times \rho N$ random matrix $\mathbf{H}$ with i.i.d. elements having zero mean and variance $1 / N$. Similarly, (11) corresponds to the $\eta$ transform of the $\mathbf{H H}^{\dagger}$ with $\mathbf{H}$ of dimension $N \times \sigma N$. Since the $\mathbf{H H}^{\dagger}$ follows the Marchenko-Pastur (MP) law given by Theorem 8.10 in Appendix A, the $\Sigma$ transforms of $\hat{\mathbf{R}}$ and $\hat{\mathbf{R}}_{1}$ can be written as [46]

$$
\Sigma_{\hat{\mathbf{R}}}(z)=\frac{1}{\rho+z}, \Sigma_{\hat{\mathbf{R}}_{1}}(z)=\frac{1}{\sigma+z}
$$

Based on the detailed analysis presented in Appendix B, we derive the following theorem for the Stieltjes transform of the $\hat{\mathbf{R}}_{\mathbf{Y}}(N)$.

Theorem 3.1: The Stieltjes transform $\mathcal{S}_{\hat{\mathbf{R}}_{\mathbf{Y}}}(z)$ of the asymptotic distribution of eigenvalues of $\frac{1}{N} \mathbf{Y} \mathbf{Y}^{H}$, where $\mathbf{Y}=\mathbf{A U} p^{1 / 2} \mathbf{B X}+\mathbf{Z}$ for arbitrary value of $p$ can be obtained for any $z \in \mathbb{C}$ by solving a polymonial 
with the following coefficients

$$
\begin{aligned}
& c_{0}=-p^{2}, \\
& c_{2}=p^{2}(\rho \sigma-1-z)-p^{3}(\rho+\sigma)+p^{4}, \\
& c_{3}=-p^{3}(\rho+\sigma)(z+1)+2 p^{2}\left(\rho \sigma-z+z p^{2}\right), \\
& c_{4}=z p^{4}(2-z)-2 z p^{3}(\rho+\sigma)-p^{2}(z-\rho \sigma), \\
& c_{5}=2 z^{2} p^{4}-z p^{3}(\rho+\sigma), \\
& c_{6}=p^{4} z^{2},
\end{aligned}
$$

where $c_{n}$ is the $n$th order coefficient of the polymonial, $\rho$ and $\sigma$ denote the compression ratio and sparsity order respectively, and $p$ is the common receive SNR of all the PU signals.

Proof: By applying multiplicative and additive free convolution properties of $\Sigma$ and $\mathrm{R}$ transforms and then using the relation (29), the polymonial in (13) is obtained. For detailed proof, see Appendix B.

The aepdf of $\hat{\mathbf{R}}_{\mathbf{Y}}$ can be obtained using Stieltjes inversion formula (35).

\section{ANALYSIS FOR VARYING POWER CASE}

Using the similar arguments as in Section III, the measured signal's sample covariance matrix for large values of $N$ can be written using the following asymptotic approximation:

$$
\lim _{N \rightarrow \infty} \hat{\mathbf{R}}_{\mathbf{Y}}(N) \approx \hat{\mathbf{R}} \mathbf{P}^{1 / 2} \hat{\mathbf{R}}_{1} \mathbf{P}^{1 / 2}+\hat{\mathbf{R}}_{\mathbf{Z}}
$$

To model the received power, uniform distribution over a certain power range would be the most suitable choice but it is analytically intractable because its transforms include logarithmic functions. To overcome this, we use modified semicircular distribution since it is symmetric around a non-zero mean and analytically tractable. The standard semicircular law is given by [46]

$$
f_{\mathrm{S}}(z)=\frac{1}{2 \pi} \sqrt{4-z^{2}},-2<z<2
$$

We extend (15) in the following form to allow for non-zero power mean and scalable DR of the power profile.

$$
f_{\mathrm{MS}}(z)=\frac{g}{2 \pi} \sqrt{4-g^{2}(z-\bar{p})^{2}}, \bar{p}-\frac{2}{g}<z<\bar{p}+\frac{2}{g} .
$$


where $\bar{p}$ denotes the mean of the considered distribution and $g$ denotes the scaling factor of the DR with $g \geq 2 / \bar{p}$. Let $[a, b]$ denote the support of the considered distribution, then the parameters $\bar{p}$ and $g$ can be expressed as: $\bar{p}=\frac{a+b}{2}$ and $g=\frac{4}{b-a}$. The DR of power variation across the carriers can be defined as: $\mathrm{DR}=10 \log \frac{b}{a}$.

Lemma 4.1: The Stieltjes transform of the modified semicircular law (16) is given by

$$
\mathcal{S}_{\mathbf{P}}(z)=\frac{1}{2} g\left[-g(z-\bar{p})+\sqrt{g^{2}(z-\bar{p})^{2}-4}\right] .
$$

Proof: By definition of the transform and integration over (16).

Lemma 4.2: The $\Sigma$ transform of the modified semicircular law (16) is given by

$$
\Sigma_{\mathbf{P}}(z)=\frac{1}{2} \frac{g\left(g c+\sqrt{g^{2} \bar{p}^{2}-8-4 z}\right)}{z} .
$$

Proof: By definition of the transform and (17).

Lemma 4.3: The Stieltjes transform of the asymptotic distribution of eigenvalues of $\hat{\mathbf{R}} \mathbf{P}^{1 / 2} \hat{\mathbf{R}} \mathbf{P}^{1 / 2}$, let us denote by $\mathbf{W}$, can be obtained for any $z \in \mathbb{C}$ by finding roots of the equation

$$
\mathcal{S}_{\mathbf{W}}(z)\left(\rho-z \mathcal{S}_{\mathbf{W}}(z)-1\right)\left(\sigma-z \mathcal{S}_{\mathbf{W}}(z)-1\right)+\frac{1}{2} g^{2} \bar{p}+\frac{1}{2} g \sqrt{g^{2} \bar{p}^{2}+4 z \mathcal{S}_{\mathbf{W}}(z)-4}=0
$$

Proof: By applying the multiplicative free convolution property of $\Sigma$ transform and using the definitions of the transforms.

Theorem 4.1: The Stieltjes transform $\mathcal{S}_{\hat{\mathbf{R}}_{\mathbf{Y}}}(z)$ of the asymptotic distribution of eigenvalues of $\frac{1}{N} \mathbf{Y} \mathbf{Y}^{H}$, where $\mathbf{Y}=\mathbf{A U P}^{1 / 2} \mathbf{B X}+\mathbf{Z}$ can be obtained for any $z \in \mathbb{C}$ by finding roots of the equation

$$
\begin{aligned}
& \mathcal{S}_{\hat{\mathbf{R}}_{\mathbf{Y}}}^{-1}(z)=z\left(\rho-z \mathcal{S}_{\mathbf{W}}(z)-1\right)\left(\sigma-z \mathcal{S}_{\mathbf{W}}(z)-1\right) \\
& \quad+\frac{1}{2} g^{2} \bar{p}+\frac{1}{2} g \sqrt{g^{2} \bar{p}^{2}+4 z \mathcal{S}_{\mathbf{W}}(z)-4}+\frac{1}{1-z},
\end{aligned}
$$

where $g$ is the scaling factor of the DR, $\rho$ and $\sigma$ denote the compression ratio and sparsity order respectively, and $\bar{p}$ is the mean SNR of the considered distribution.

Proof: By applying multiplicative and additive free convolution properties of $\Sigma$ and $\mathrm{R}$ transforms and then using the definitions of the transforms. For detailed proof, see Appendix C.

Remark 4.1: The closed form of (20) is not specifically provided in this paper because it includes many terms which provide no further insight. In practice, we can just solve this equation with a mathematical software in order to find the Stieltjes transform and subsequently the aepdf. 


\section{ANALYSIS UNDER CORRELATED SCENARIO}

In this case, we consider correlation across the non-zero entries of the multiple measurement vectors. To model this scenario, we consider the one-sided noise correlation model as in $[24,55]$. We model the correlated sparse signal matrix as: $\hat{\mathbf{S}}=\Theta^{1 / 2} \mathbf{S}$, where $\Theta^{1 / 2} \Theta^{1 / 2}=\Theta=\mathbb{E}\left[\hat{\mathbf{S}} \hat{\mathbf{S}}^{H}\right]$. To ensure that $\Theta$ does not affect the received signal power, the normalization $(1 / N) \operatorname{trace}\{\boldsymbol{\Theta}\}=1$ is considered. We model the components of the covariance matrix $\Theta$ using an exponential correlation model given by [50];

$$
\theta_{i j} \sim \begin{cases}\varsigma^{(j-i)}, & i \leq j \\ \left(\varsigma^{(i-j)}\right)^{*}, & i>j\end{cases}
$$

where $\theta_{i j}$ is the $(i, j)$ th element of $\Theta$ and $\varsigma \in \mathbb{C}$ is the correlation coefficient with $|\varsigma| \leq 1$. A similar correlation model has been applied in [56] for sparse support recovery in the presence of correlated multiple measurements. Using the correlation model in (21), the observation model is given by (6).

For correlation analysis in this paper, we consider that the correlation matrix $\Theta$ follows the tilted semicircular law ${ }^{2}$ as in [51], which is given by

$$
f_{\boldsymbol{\Theta}}(z)=\frac{1}{2 \pi \mu z^{2}} \sqrt{\left(\frac{z}{\delta_{1}}-1\right)\left(1-\frac{z}{\delta_{2}}\right)}
$$

where $\left[\delta_{1}, \delta_{2}\right]$ defines the support of the distribution and $\mu=\frac{\left(\sqrt{\delta_{2}}-\sqrt{\delta_{1}}\right)}{4 \delta_{1} \delta_{2}}$ with $\mu>0$. The parameter $\mu$ controls the degree of correlation and varies the support of the distribution. The Signal Condition Number (SCN) of the $N \times N$ correlation matrix $\Theta$ is related to $\mu$ as: $\mu=\frac{\mathrm{SCN}-1}{\mathrm{SCN}+1}$. As stated in [51], the parameter $\mu$ is related to $\varsigma$ as: $\mu=\frac{\varsigma^{2}}{1-\varsigma^{2}}$. In this work, we are interested in carrying out SOE for different levels of correlation across the non-zero entries of the MMV model. For this purpose, we need the aepdf of $\hat{\mathbf{R}}_{\mathbf{Y}}(N)$ under the correlated scenario. Using the similar arguments as in earlier sections, the measured signal's sample covariance matrix for large values of $N$ can be written using the following asymptotic approximation

$$
\lim _{N \rightarrow \infty} \hat{\mathbf{R}}_{\mathbf{Y}}(N) \approx p \hat{\mathbf{R}} \Theta^{1 / 2} \hat{\mathbf{R}}_{1} \Theta^{1 / 2}+\hat{\mathbf{R}}_{\mathbf{Z}}
$$

Theorem 5.1: The Stieltjes transform $\mathcal{S}_{\hat{\mathbf{R}}_{\mathbf{Y}}}(z)$ of the asymptotic distribution of eigenvalues of $\frac{1}{N} \mathbf{Y} \mathbf{Y}^{H}$ where $\mathbf{Y}=\mathbf{A} \mathbf{U} \boldsymbol{\Theta}^{1 / 2} \sqrt{p} \mathbf{B X}+\mathbf{Z}$ can be obtained for any $z \in \mathbb{C}$ by solving a polymonial with the

\footnotetext{
${ }^{2}$ It can be noted that this law closely approximates the exponential correlation model given by (21).
} 
following coefficients

$$
\begin{aligned}
& c_{0}=p \mu(1+p \mu), \\
& c_{1}=2 p \mu\left(z p \mu-p^{2}\right)+p^{2}(\mu(\rho+\sigma)-1)+p \mu(z-\rho \sigma+1), \\
& c_{2}=(\rho+\sigma)\left(p^{2} \mu+z p^{2} \mu-p^{3}\right)+p \rho \sigma(p-2 \mu)+2 z p \mu\left(p \mu+1-2 p^{2}\right)-p^{2}\left(1+z-z^{2} \mu^{2}\right), \\
& c_{3}=2 z p^{2}\left(\mu(\rho+\sigma)-z \mu(p-\mu)+p^{2}-2 p \mu-1\right)-p^{3}(z+1)(\rho+\sigma)+p(\rho \sigma(2 p-\mu)+z \mu), \\
& c_{4}=z p^{2} \mu(\rho+\sigma)-2 z p^{3}(\rho+1)+p^{2}\left(z^{2}\left(\mu^{2}-4 p \mu+p^{2}\right)+\rho \sigma-z+2 z p^{2}\right), \\
& c_{5}=2 z^{2} p^{3}(p-\mu)-z p^{3}(\rho+\sigma), \\
& c_{6}=z^{2} p^{4},
\end{aligned}
$$

where $c_{n}$ is the $n$th order coefficient of the polymonial, $\rho$ and $\sigma$ denote the compression ratio and sparsity order respectively, $\mu$ defines the correlation degree of $\Theta$, and $p$ is the common receive SNR of all the PU signals.

Proof: By definitions of the transforms and using multiplicative and additive free convolution properties of $\Sigma$ and R transforms respectively. For detailed proof, see Appendix D.

\section{Proposed Compressive Sparsity Order Estimation Method}

The SOE is the process of identifying the number of nonzero elements of a sparse vector and does not need to have the exact knowledge of their amplitudes or positions. The proposed compressive sparsity order estimation can be applicable in general settings. In this paper, this problem is mainly motivated by wideband $\mathrm{CR}$ scenarios where compressive SOE is the main issue for determining the suitable sampling rate at the receiver. To determine the suitable sampling rate at the $\mathrm{CR}$ receiver, most existing CS literature implicitly assumes that the sparsity order of the considered wideband spectrum is known beforehand. However, in practical CR applications, the actual sparsity order corresponds to the instantaneous spectrum occupancy of wireless users and it varies dynamically as the spectrum occupancy changes. Hence, the sparsity order is often unknown and only its upper bound can be measured based on the maximum spectrum utilization observed statistically over a time period. In practice, the determination of the sampling rate based on the upper bound can cause unnecessarily high sensing cost since the sampling rate depends on the sparsity order [23]. From the above discussion, it can be noted that it is crucial to adapt the sampling rate in accordance to the sparsity variation of the spectrum occupancy and thus tracking the instantaneous 
sparsity order is an important issue. In this context, we propose an eigenvalue-based blind SOE method which is based on the maximum eigenvalue of the measured signal's sample covariance matrix.

Based on the polymonials of the Stieltjes transforms specified in the above sections, the support range of the corresponding aepdf is obtained using $(35)^{3}$. For convenience, a lookup table (Table I) is provided in order to illustrate the SOE method in the considered scenarios (see Section VII). In the lookup table, we present the maximum eigenvalues of $\hat{\mathbf{R}}_{\mathbf{Y}}$ for the considered three different cases and the corresponding values of $\sigma$. For sparsity order estimation purpose, we select $K$ number of sampling slots and calculate $\bar{\lambda}_{\max }=\mathbb{E}_{K}\left[\lambda_{\max }\left(\hat{\mathbf{R}}_{\mathbf{Y}}(N)\right)\right]$ over these slots. Subsequently, $\bar{\lambda}_{\max }$ is compared with the values of $\lambda_{\max }$ stored in the lookup table and the corresponding value of $\sigma$ is obtained. Further, for any intermediate values of $\bar{\lambda}_{\text {max }}$, a suitable interpolation method can be applied for estimating the corresponding value of $\sigma$. To clarify the above process, we include procedures for lookup table formation and sparsity order estimation considering the constant power scenario (Section III) below.

In all the considered cases, the parameter $\rho$ is assumed as an operating parameter of the CR sensing module and its value depends on how much compressed measurements we want to carry out in order to reduce the hardware costs at the expense of some estimation error. The Stieltjes transform of $\hat{\mathbf{R}}_{\mathbf{Y}}$ for the constant power case is calculated using polymonial (13). In this case, the value of $p$ is assumed to be known and in practice, its value can be obtained by using SNR estimation techniques like in [24]. Since we know the value of $\rho$ and $p$ in (13), we can estimate the value of $\sigma$ by sensing the $\bar{\lambda}_{\max }$ of $\hat{\mathbf{R}}_{\mathbf{Y}}$, where $\mathbf{Y}=\mathbf{A} \mathbf{U} p^{1 / 2} \mathbf{B X}+\mathbf{Z}$, obtained using (13) and (35).

Similarly, the Stieltjes transform for the varying power case is obtained using equation (20). In this case, the support of the considered power distribution i.e., $[a, b]$ is assumed to be known and based on this support, the values of $g$ and $\bar{p}$ can be calculated as mentioned in Section IV. As we know the value of $g, \bar{p}$ and $\rho$ in (20), we can estimate the sparsity order i.e, $\sigma$ by evaluating $\bar{\lambda}_{\max }$ of $\hat{\mathbf{R}}_{\mathbf{Y}}$, where $\mathbf{Y}=\mathbf{A} \mathbf{U} \mathbf{P}^{1 / 2} \mathbf{B X}+\mathbf{Z}$, obtained using (20) and (35).

In the similar way, the Stieltjes transform for the correlated case is obtained using the polymonial (24). In this case, the value of $\varsigma$ is assumed to be known and in practice, its value can be found using different noise correlation models such as in $[24,56]$ or measurement methods. Based on the value of $\varsigma$, the value of $\mu$ can be calculated as mentioned in Section V. Since we know the value of $p, \rho$ and $\mu$ in (24), we can

\footnotetext{
${ }^{3}$ We select the imaginary root which complies with the definition and properties of Stieltjes transform (See Appendix A).
} 
estimate the value of $\sigma$ by calculating the maximum eigenvalue of $\hat{\mathbf{R}}_{\mathbf{Y}}$, where $\mathbf{Y}=\mathbf{A} \mathbf{U} \Theta^{1 / 2} \sqrt{p} \mathbf{B X}+\mathbf{Z}$, obtained using (24) and (35).

For example, for Scenario I, if the value of $\bar{\lambda}_{\max }$ of the $\hat{\mathbf{R}}_{\mathbf{Y}}$ is 6.67 under compressive measurement scenario with $\rho=0.8$, it can be estimated that the sparsity order of the considered wideband spectrum is 0.5 . Based on this estimated sparsity order, we could potentially fix the optimum sampling rate of the CS acquisition system in order to reduce the sensing hardware cost. In Section VII, we provide the normalized SOEE versus sparsity order plots to evaluate the performance of this estimation technique.

\section{Procedure for lookup table formation}

1) Select the operating parameter $\rho$.

2) Select the value of $p$ based on SNR estimation techniques like in [24].

3) Evaluate $\mathcal{S}_{\hat{\mathbf{R}}_{\mathbf{Y}}}(z)$ using (13).

4) Find $\lambda_{\max }\left(\hat{\mathbf{R}}_{\mathbf{Y}}\right)$ using (35).

5) For each value of $\sigma$ in $(0,1)$, repeat steps 3 and 4.

6) Store all $\lambda_{\max }\left(\hat{\mathbf{R}}_{\mathbf{Y}}\right)$ and corresponding $\sigma$ e.g., Table I (Scenario I).

Procedure for sparsity order estimation

1) Select the number of sampling slots $K$.

2) Calculate instantaneous $\hat{\mathbf{R}}_{\mathbf{Y}}(N)=\frac{1}{N} \mathbf{Y} \mathbf{Y}^{H}$ in each sampling slot.

3) Calculate $\bar{\lambda}_{\max }=\mathbb{E}_{K}\left[\lambda_{\max }\left(\hat{\mathbf{R}}_{\mathbf{Y}}(N)\right)\right]$ over $K$ number of sampling slots.

4) Find $\sigma$ corresponding to $\bar{\lambda}_{\max }$ from the lookup table.

5) Use suitable interpolation for any intermediate value of $\bar{\lambda}_{\max }$.

\section{NUMERICAL RESULTS}

To evaluate the performance of the proposed SOE method for the considered scenarios, the metric normalized SOE Error (SOEE) is used, which is expressed in terms of root mean square error normalized to the true sparsity order and defined by

$$
\mathrm{SOEE}=\frac{\sqrt{\mathbb{E}\left[(\hat{\sigma}-\sigma)^{2}\right]}}{\sigma},
$$

where $\hat{\sigma}$ is the estimated sparsity order with the proposed method and $\sigma$ is the actual sparsity order. In our numerical results, we run $10^{3}$ number of Mote Carlo realizations in order to obtain the value of 
normalized SOEE in (25) and each Monte Carlo realization includes steps (2) and (3) from the sparsity order estimation procedure specified in Section VI.

The total error in our estimation process results from the two sources. The first error comes from the randomness of the maximum eigenvalue which is used as an input parameter for the estimator and the second error is the residual error resulting from the interpolation process. In practice, the second error can be minimized by having a dense look up table and the first error can be minimized by increasing the number of sensing slots over which the mean eigenvalue i.e., $\bar{\lambda}_{\max }$ is calculated. However, in practice, the number of sampling slots are limited due to the limited sampling time required to capture the dynamicity of the time varying sparsity order. If the spectrum occupancy changes too rapidly i.e., the sparsity order varies at a faster rate, the number of sampling slots should be small. To take this fact into account, we use only $K=30$ number of sampling slots in our results.

In the following subsections, we present numerical results for SOE for the considered three different scenarios. In the numerical results, we compare the performance of the proposed SOEE technique in compressive and full measurement cases. The compressive measurement case corresponds to the signal model given by (2) while the full measurement case for the constant power scenario corresponds to the following signal model $\mathbf{Y}=\sqrt{p} \mathbf{B X}+\mathbf{Z}$ and the following sample covariance matrix $\hat{\mathbf{R}}_{\mathbf{Y}}=p \hat{\mathbf{R}}_{1}+\hat{\mathbf{R}}_{\mathbf{Z}}$. It should be noted that the theoretical analysis for the full measurement case has already been carried out in [24]. From practical perspectives, the difference between full measurement and compressive measurement cases is that the former considers all the measurements across the carriers whereas the later case considers the sparse linear combinations of carrier measurements.

\section{A. Constant Power Scenario}

To validate our theoretical analysis presented in Section III, we plot the theoretical and simulated eigenvalue distributions of $\hat{\mathbf{R}}_{\mathbf{Y}}(N)$ in Fig. 1 with parameters $\rho=0.8, \sigma=0.6, N=100, \mathrm{SNR}=0 \mathrm{~dB}$. The theoretical aepdf in this case was obtained by solving the polymonial given by (13) and using the Stieltjes inversion formula in (35). From the figure (Fig. 1), it can be noted that the theoretical curve perfectly matches with the simulated one.

For sparsity order estimation purpose, we provide a lookup table (Table I, Scenario I), where the maximum eigenvalues of $p \hat{\mathbf{R}} \hat{\mathbf{R}}_{1}+\hat{\mathbf{R}}_{\mathbf{Z}}$ are provided for different values of $\sigma$ for compressive and full measurement cases. The value of $\sigma$ can be estimated using this table based on the SOE method described 


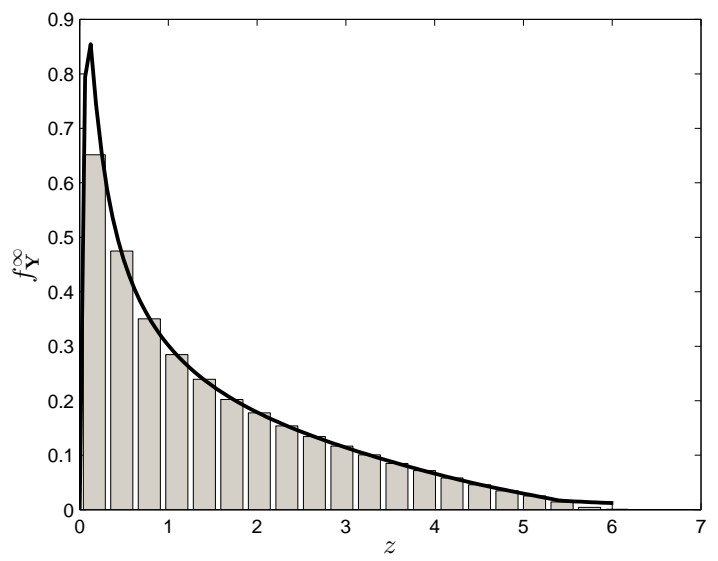

Fig. 1: Theoretical and simulated eigenvalue distribution of $\hat{\mathbf{R}}_{\mathbf{Y}}(N)$, where $\mathbf{Y}=\mathbf{A} \mathbf{U}_{\sqrt{p}} \mathbf{B X}+\mathbf{Z}$ $(\rho=0.8, \sigma=0.6, N=100, \mathrm{SNR}=0 \mathrm{~dB})$

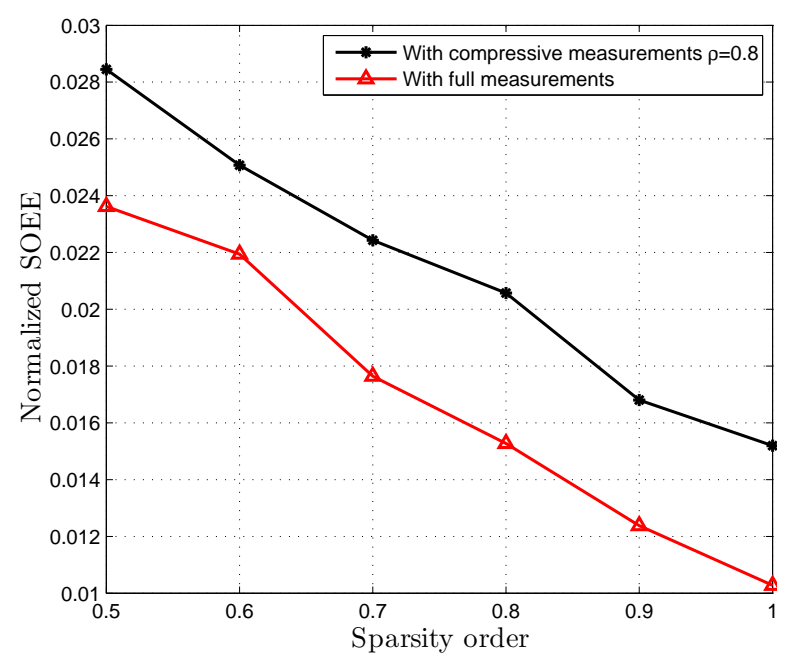

Fig. 2: Normalized SOEE versus sparsity order with compressive and full measurements for constant received power scenario $(\mathrm{SNR}=2 d B, N=100)$

in Section VI. For example, if the value of $\bar{\lambda}_{\max }$ is 6.05 for the compressive measurement case, it can be estimated that the sparsity order of the occupancy of the considered wideband spectrum is $40 \%$.

Figure 2 presents the normalized SOEE versus sparsity order for compressive and full measurement cases for $S N R=2 \mathrm{~dB}$. From this figure, it can be noted that the normalized SOEE is higher for the compressive case than for the full measurement case. For SNR $=2 \mathrm{~dB}$ as shown in Fig. 2, the normalized SOEE for the compressive case is slightly less than $2.9 \%$ and for the full measurement case is nearly about $2.4 \%$ at the sparsity order of 0.5 . On the other hand, the advantage is that we have used the compression of $80 \%$ i.e., $20 \%$ saving can be achieved in terms of hardware resources, which is a considerable gain. Furthermore, Fig. 3 presents the normalized SOEE versus SNR considering a fixed sparsity order of 0.6. 
Our simulation results show that at lower values of SNR, the compressive case performs better than the full measurement case in terms of the normalized SOEE (below the SNR value of $-0.5 \mathrm{~dB}$ in Fig. 3). An intuitive explanation is that in the full measurement case, the contribution of the noise in the aepdf becomes dominant with a faster rate compared to the compressed measurement case.

Figure 4 presents the estimation error in terms of the normalized SOEE versus compression ratio $\rho$ for the SNR value of $2 \mathrm{~dB}$. In this simulation settings, the value of $\sigma$ was considered as 0.6 and the estimation error for each $\rho$ was calculated by interpolating the value of $\bar{\lambda}_{\max }$ with the provided set of the values of $\sigma$ and $\lambda_{\max }$ for the considered value of $\rho$. It can be noted that $\rho=1$ corresponds to no-compression and the estimation error in terms of normalized SOEE increases as $\rho$ decreases (Fig. 4) i.e., with the increase in the compression. It can be depicted that by using the smaller value of compression ratio $\rho$, we are saving sampling resources in one hand but on the other hand, we have to sacrifice some estimation performance. Therefore, in practice, there exists a tradeoff between the number of measurements (hardware cost) and the estimation performance.

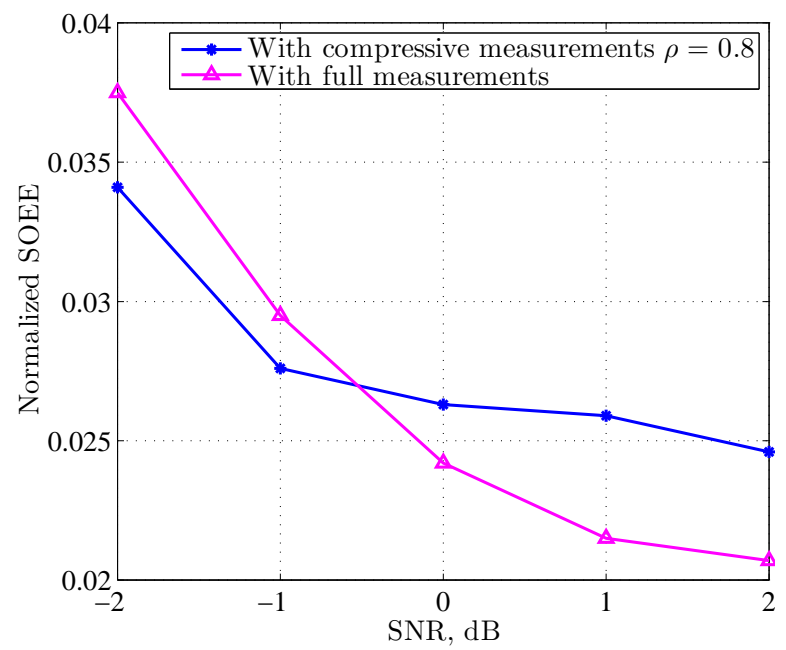

Fig. 3: Normalized SOEE versus SNR with compressive and full measurements for constant received power scenario $(\sigma=0.6, N=100)$

\section{B. Varying Power Scenario}

Under this scenario, received power levels across the carriers within the considered wideband spectrum vary but they are assumed to follow a known distribution as mentioned in Section IV. The main advantage of the applied approach is that we do not need to have information of all the individual channel powers in order to estimate the sparsity order. In order to validate the theoretical analysis presented in Section IV, we provide the theoretical and simulated distributions of $\hat{\mathbf{R}}_{\mathbf{Y}}(N)$ in Fig. 5. The theoretical curve was 


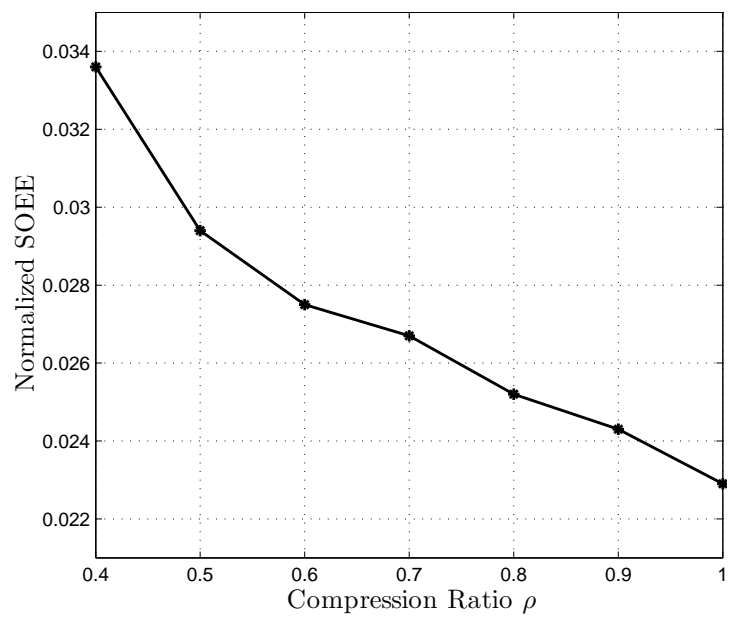

Fig. 4: Normalized SOEE versus compression ratio $\rho(\mathrm{SNR}=2 \mathrm{~dB}, \sigma=0.6, N=100)$

obtained by evaluating equation (20) and using the Stieltjes inversion formula in (35). It can be noted that the theoretical and simulated results match perfectly.

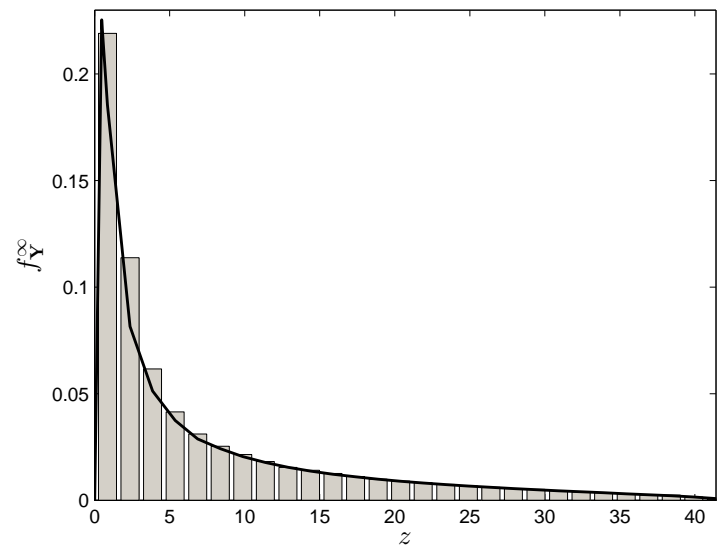

Fig. 5: Theoretical and simulated eigenvalue distribution of $\hat{\mathbf{R}}_{\mathbf{Y}}(N)$, where $\mathbf{Y}=\mathbf{A} \mathbf{U} \mathbf{P}^{1 / 2} \mathbf{B X}+\mathbf{Z}$ $(\mathrm{DR}=6.02 d B, N=100, \bar{p}=7.78 \mathrm{~dB})$

TABLE I: Lookup table for sparsity order estimation for the considered scenarios $(\rho=0.8)$

\begin{tabular}{|c|c|c|c|c|c|c|}
\hline $\begin{array}{c}\text { Scenarios } \\
\text { Sparsity Level }\end{array}$ & $\begin{array}{c}\text { I } \\
\text { SNR=2 dB } \\
\text { Compressive }\end{array}$ & $\begin{array}{c}\text { I } \\
\text { SNR=2 dB } \\
\text { Full }\end{array}$ & $\begin{array}{c}\text { II } \\
\mathrm{DR}=6.02 \mathrm{~dB} \\
\text { Compressive }\end{array}$ & $\begin{array}{c}\text { II } \\
\mathrm{DR}=6.02 \mathrm{~dB} \\
\text { Full }\end{array}$ & $\begin{array}{c}\text { III } \\
\mathrm{SCN}=4 \mathrm{SNR}=0 \mathrm{~dB} \\
\text { Compressive }\end{array}$ & $\begin{array}{c}\text { III } \\
\mathrm{SCN}=4 \underset{\mathrm{SNR}}{\text { Full }}=0 \mathrm{~dB}\end{array}$ \\
\hline 1 & 9.63 & 7.44 & 12.80 & 10.19 & 6.93 & 5.86 \\
\hline 0.9 & 9.03 & 7.14 & 11.80 & 9.58 & 6.53 & 5.65 \\
\hline 0.8 & 8.41 & 6.83 & 11.03 & 9.08 & 6.17 & 5.44 \\
\hline 0.7 & 7.85 & 6.53 & 10.22 & 8.60 & 5.80 & 5.24 \\
\hline 0.6 & 7.26 & 6.21 & 9.36 & 8.08 & 5.43 & 5.02 \\
\hline 0.5 & 6.67 & 5.88 & 8.42 & 7.44 & 5.08 & 4.81 \\
\hline 0.4 & 6.05 & 5.52 & 7.51 & 6.84 & 4.74 & 4.60 \\
\hline 0.3 & 5.44 & 5.15 & 6.63 & 6.24 & 4.43 & 4.40 \\
\hline 0.2 & 4.85 & 4.76 & 5.66 & 5.57 & 4.17 & 4.20 \\
\hline 0.1 & 4.25 & 4.31 & 4.69 & 4.80 & 3.96 & 4.00 \\
\hline 0 & 3.79 & 3.79 & 3.79 & 3.79 & 3.79 & 3.79 \\
\hline
\end{tabular}




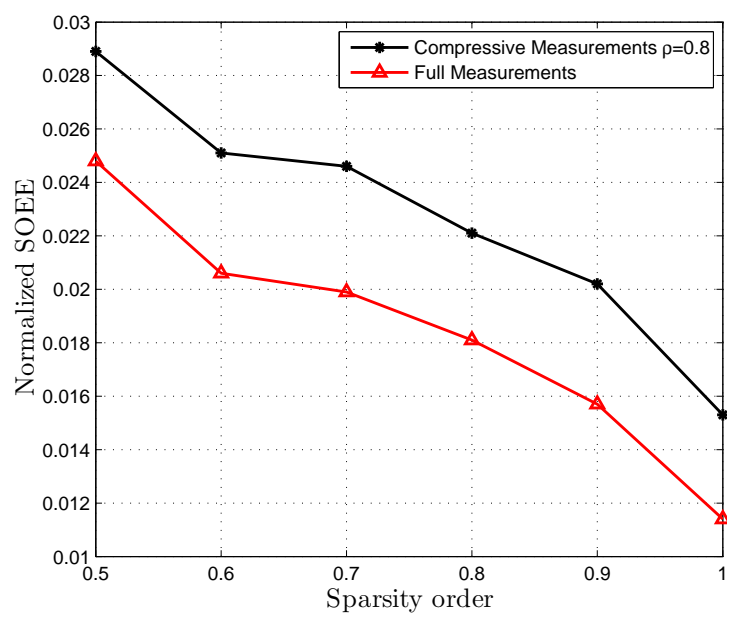

Fig. 6: Normalized SOEE versus sparsity order with compressive and full measurements for varying power scenario $(\mathrm{DR}=6.02 d B, N=100, \bar{p}=7.78 \mathrm{~dB})$

In order to estimate the sparsity order under this scenario, the lookup table (Table I, Scenario II) can be used, where the maximum eigenvalues of $\hat{\mathbf{R}} \mathbf{P} \hat{\mathbf{R}}_{1}+\hat{\mathbf{R}}_{\mathbf{Z}}$ are provided for different values of $\sigma$ for compressive and full measurement cases. For example, if the value of $\bar{\lambda}_{\max }$ is 9.36 for the compressive case, it can be estimated that the sparsity order of the occupancy of the considered wideband spectrum is $60 \%$.

Figure 6 presents the comparison of the normalized SOEE versus sparsity order for full and compressive measurement cases with parameters $(\mathrm{DR}=6.02 \mathrm{~dB}, N=100, \bar{p}=7.78 \mathrm{~dB})$. From the figure, it can be noted that sparsity order up to 0.5 can be estimated with less than $2.9 \%$ estimation error for the compressive case and with less than $2.5 \%$ estimation error for the full measurement case. Furthermore, it can be noted that we have to tolerate a small amount of additional error while using compressive measurements. On the other hand, we are saving $20 \%$ hardware resources using $20 \%$ less measurements.

\section{Correlated MMV Scenario}

Under this scenario, we consider correlated multiple measurement vectors as mentioned in Section V. To validate the theoretical analysis presented in Section V, we present the theoretical and simulated eigenvalue distributions of the measured signal's covariance matrix given by (23) in Fig. (7) with parameters ( $\rho=$ $0.8, \sigma=0.6, N=100, \mathrm{SCN}=4)$. The theoretical plot was obtained by solving the polymonial (24) and using the Stieltjes inversion formula in (35). From the figure, it can be noted that the theoretical and simulated results match perfectly. In order to estimate the sparsity order, we can use the $\lambda_{\max }$ of the aepdf obtained from the Stieltjes transform given by polymonial (24). Since we assume the value of $\rho, \mu$ to 
be known in (24), the value of $\sigma$ can be estimated based on the procedure described in Section VI. For convenience, we provide lookup table (Table I, Scenario III).

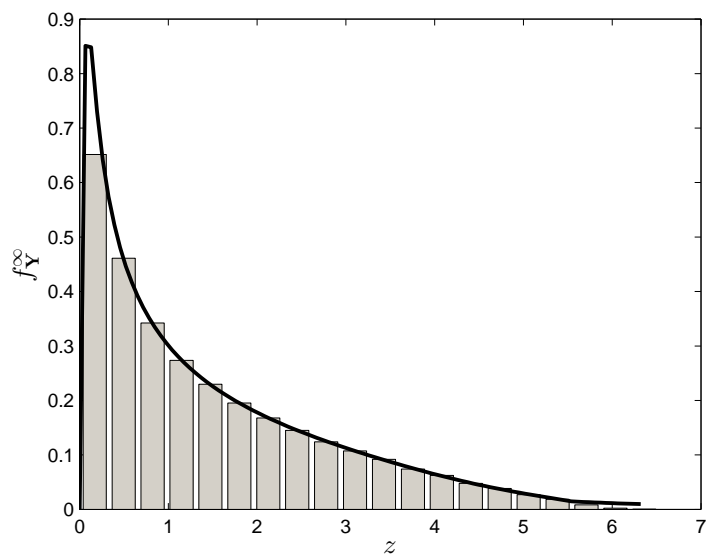

Fig. 7: Theoretical and simulated eigenvalue distribution of $\hat{\mathbf{R}}_{\mathbf{Y}}(N)$, where $\mathbf{Y}=\mathbf{A} \mathbf{U} \Theta^{1 / 2} \sqrt{p} \mathbf{B X}+\mathbf{Z}$ $(\rho=0.8, \sigma=0.6, N=100, \mathrm{SCN}=4, \mathrm{SNR}=0 \mathrm{~dB})$

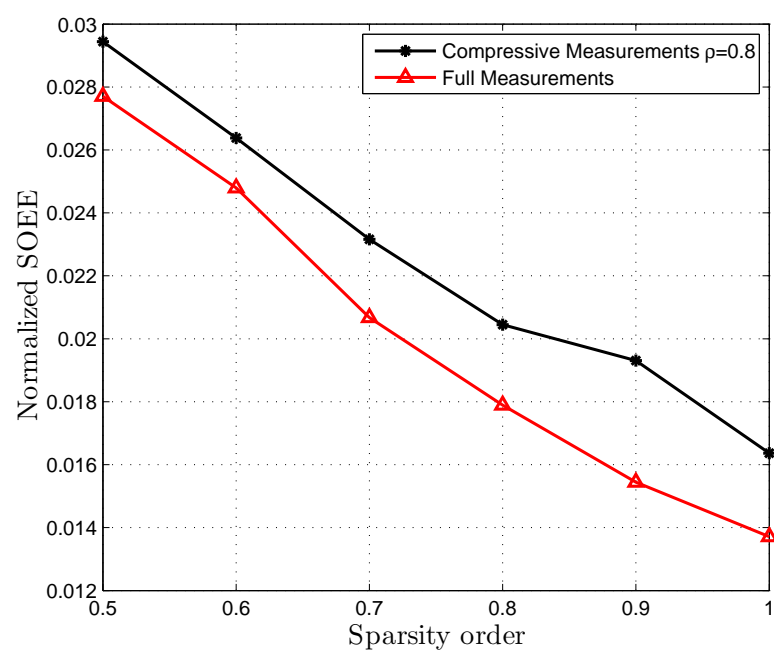

Fig. 8: Normalized SOEE versus sparsity order with compressive and full measurements for correlated and uncorrelated scenarios $(\mathrm{SCN}=4, N=100, \mathrm{SNR}=0 \mathrm{~dB})$

Figure 8 shows the normalized SOEE versus sparsity order comparison for compressive and full measurement cases for the correlated MMV scenario with parameters $(\mathrm{SCN}=4, N=100, \mathrm{SNR}=0$ dB). From the figure, It can be noted that the full measurement case performs better than the compressive measurement case with $\rho=0.8$. The sparsity order can be estimated with less than $3 \%$ for the compressive measurement case and with less than $2.8 \%$ for the full measurement case up to the sparsity order of 0.5 (as depicted in Fig. 8). As in previous scenarios, it can be concluded that we need to sacrifice some amount of performance in terms of estimation error in order to save the sensing hardware resources. 


\section{CONCLUSION}

In this paper, a novel technique has been proposed for estimating the sparsity order of spectrum occupancy within a wideband spectrum in the context of a wideband CR. First, the theoretical expressions for aepdf of the measured signal's covariance matrix have been derived for three different scenarios using RMT-based methods. More specifically, the following different scenarios have been considered: (i) constant received power scenario, (ii) varying received power scenario, and (iii) correlated scenario with the correlated multiple measurement vectors. Then the performance of the proposed method was evaluated in terms of the normalized SOEE for the considered scenarios. It can be concluded that the proposed technique can reliably estimate the sparsity order for a range of scenarios even with compressive measurements. Further, it has been noted that there exists a tradeoff between the hardware sensing cost and the estimation error while using compressive measurements. In our future work, we plan to apply the proposed compressive SOE technique for adaptive CS at the CR receiver and to further explore blind signal processing techniques for estimating the positions of vacant carriers using compressive measurements.

\section{APPENDIX A}

\section{Random Matrix Theory Preliminaries}

Let $F_{\mathbf{X}}(x)$ be the eigenvalue probability density function of a matrix $\mathbf{X}$.

Theorem 8.1: The Stieltjes transform $\mathcal{S}_{\mathbf{X}}(z)$ of a positive semidefinite matrix $\mathbf{X}$ is defined by [46]

$$
\mathcal{S}_{\mathbf{X}}(z)=\mathbb{E}\left[\frac{1}{\mathbf{X}-z}\right]=\int_{-\infty}^{\infty} \frac{1}{\lambda-z} d F_{\mathbf{X}}(\lambda) .
$$

Theorem 8.2: The $\eta$ transform of a positive semidefinite matrix $\mathbf{X}$ can be defined as [46]

$$
\eta_{\mathbf{X}}(\gamma)=\mathbb{E}\left[\frac{1}{1+\gamma \mathbf{X}}\right]
$$

where $\gamma$ is a nonnegative real number and thus $0 \leq \eta_{\mathbf{X}}(\gamma) \leq 1$.

Theorem 8.3: The $\Sigma$ transform of the density of eigenvalues of $\mathbf{X}$ can be defined using $\eta$ transform in the following way [46]

$$
\Sigma_{\mathbf{X}}(z)=-\frac{1+z}{z} \eta_{\mathbf{X}}^{-1}(1+z)
$$

Theorem 8.4: The $\mathrm{R}$ transform is related to the inverse of Stieltjes transform as [46]

$$
\mathcal{R}_{\mathbf{X}}(z)=\mathcal{S}_{\mathbf{X}}^{-1}(-z)-\frac{1}{z}
$$


Theorem 8.5: For a Wishart random matrix $\mathbf{X}$, the $\mathrm{R}$ transform of the density of eigenvalues of $\mathrm{X}$ is defined as [46]

$$
\mathcal{R}_{\mathbf{X}}(z)=\frac{\beta}{1-z}
$$

For any $a>0$,

$$
\mathcal{R}_{a \mathbf{X}}(z)=a \mathcal{R}_{\mathbf{X}}(a z)
$$

Theorem 8.6: For a Wishart random matrix $\mathbf{X}$, the $\Sigma$ transform of the density of eigenvalues of $\mathbf{X}$ is defined as [46]

$$
\Sigma_{\mathbf{X}}(z)=\frac{1}{z+\beta}
$$

Theorem 8.7: The $\eta$ transform of the density of eigenvalues of $\mathbf{X}$ is related to the Stieltjes transform by the following relation [46]

$$
\eta_{\mathbf{X}}(z)=\frac{\mathcal{S}_{\mathbf{X}}\left(-\frac{1}{z}\right)}{z}
$$

Theorem 8.8: The Stieltjes transform of the semicircular law (15) is given by [52]

$$
\mathcal{S}(z)=\frac{1}{2}\left[-z+\sqrt{z^{2}-4}\right]
$$

Theorem 8.9: The aepdf of $\mathrm{X}$ is obtained by determining the imaginary part of the Stieltjes transform $\mathcal{S}_{\mathbf{X}}$ for real arguments in the following way.

$$
f_{\mathbf{X}}(x)=\lim _{y \rightarrow 0^{+}} \frac{1}{\pi} \operatorname{Im}\left\{\mathcal{S}_{\mathbf{X}}(x+j y)\right\} .
$$

Theorem 8.10: [46] Consider an $M \times N$ matrix $\mathbf{F}$ whose entries are independent zero-mean complex (or real) random variables with variance $\frac{1}{N}$ and fourth moments of order $O\left(\frac{1}{N^{2}}\right)$. As $M, N \rightarrow \infty$ with $\frac{N}{M} \rightarrow \beta$, the empirical distribution of the eigenvalues of $\frac{1}{N} \mathbf{F F}^{H}$ converges almost surely to a non-random limiting distribution with density given by

$$
f_{\beta}(\lambda)=(1-\beta)^{+} \delta(\lambda)+\frac{\sqrt{(\lambda-a)^{+}(b-\lambda)^{+}}}{2 \pi \beta \lambda},
$$

where $a=(1-\sqrt{\beta})^{2}, b=(1+\sqrt{\beta})^{2}, \delta($.$) is a Dirac delta function and (1-\beta)^{+} \delta(\lambda)$ represents the cardinality of zero eigenvalues which can occur if $M>N$. The parameters $a$ and $b$ define the support of the distribution and correspond to $\lambda_{\min }$ and $\lambda_{\max }$ respectively and the ratio $b / a$ defines the SCN of $\frac{1}{N} \mathbf{F F}^{H}$. The above limiting distribution is the MP law with ratio index $\beta$. 


\section{Proof of Theorem 3.1}

Since $\hat{\mathbf{R}}$ and $\hat{\mathbf{R}}_{1}$ are independent Wishart matrices, they are asymptotically free [46]. As a result, the combined aepdf of the term $\hat{\mathbf{R}} \hat{\mathbf{R}}_{1}$ in (7) can be obtained by applying multiplicative free convolution property of $\Sigma$ transform in the following way [53]

$$
\Sigma_{\hat{\mathbf{R}} \hat{\mathbf{R}}_{1}}(z)=\Sigma_{\hat{\mathbf{R}}}(z) \cdot \Sigma_{\hat{\mathbf{R}}_{1}}(z) .
$$

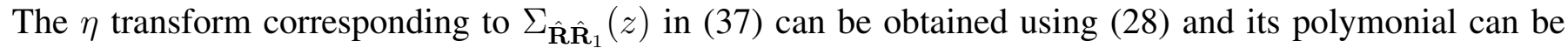
written as

$$
z(\eta(z)+1)(\eta(z)+\rho)(\eta(z)+\sigma)-\eta(z)
$$

Then using the relation between $\eta$ and Stieltjes transform given by (33), the polymonial for Stieltjes transform of the asymptotic distribution of the eigenvalues of the product of $\hat{\mathbf{R}}$ and $\hat{\mathbf{R}}_{1}$ can be written as

$$
z^{2} \mathcal{S}^{3}(z)+z(2-\rho-\sigma) \mathcal{S}^{2}(z)+(-z+(\rho-1)(\sigma-1)) \mathcal{S}(z)-1=0 .
$$

Let $\mathcal{R}_{\hat{\mathbf{R}}_{c}}$ be the $\mathrm{R}$ transform of the product term $\hat{\mathbf{R}} \hat{\mathbf{R}}_{1}$ and is calculated using (39) and (29), which is given by

$$
\mathcal{R}_{\hat{\mathbf{R}}_{c}}(z)=\frac{-(1 / 2)\left(z \rho+z \sigma-1+\sqrt{\left(z^{2} \rho^{2}-2 z^{2} \rho \sigma-2 z \rho+z^{2} \sigma^{2}-2 z \sigma+1\right)}\right)}{z^{2}} .
$$

Subsequently, the $\mathbf{R}$ transform of $p \hat{\mathbf{R}} \hat{\mathbf{R}}_{1}$ in the second term of (7) becomes $p \mathcal{R}_{\hat{\mathbf{R}}_{c}}(p z)$. Since the term $\hat{\mathbf{R}}_{\mathbf{Z}}$ in (7) follows the MP distribution, the $\mathrm{R}$ transform of $\hat{\mathbf{R}}_{\mathbf{Y}}$ using additive free convolution property can be written as

$$
\mathcal{R}_{\hat{\mathbf{R}}_{\mathbf{Y}}}(z)=p \mathcal{R}_{\hat{\mathbf{R}}_{c}}(p z)+\mathcal{R}_{\hat{\mathbf{R}}_{\mathbf{Z}}}(z)
$$

Finally, the polymonial for the Stieltjes transform of the density of $\hat{\mathbf{R}}_{\mathbf{Y}}$ in (13) is obtained using (29).

\section{APPENDIX C}

\section{Proof of Theorem 4.1}

Since $\mathbf{P}$ is a square matrix, $\mathbf{P}^{1 / 2} \hat{\mathbf{R}}_{1} \mathbf{P}^{1 / 2}$ and $\mathbf{P} \hat{\mathbf{R}}_{1}$ have identical eigenvalues [46]. Therefore, the $\Sigma$ transform of the term $\hat{\mathbf{R}} \mathbf{P}^{1 / 2} \hat{\mathbf{R}}_{1} \mathbf{P}^{1 / 2}$ in (14) can be written as

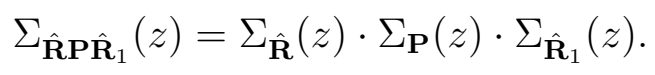


Since $\hat{\mathbf{R}}$ and $\hat{\mathbf{R}}_{1}$ follow the MP distribution, their $\Sigma$ transforms are given by (12) and the $\Sigma$ transform of $\mathbf{P}$ is given by (18). Subsequently, using (42), the following expression can be written.

$$
\Sigma_{\hat{\mathbf{R}} \mathbf{P} \hat{\mathbf{R}}_{1}}(z)=\frac{1}{\rho+z} \cdot \frac{1}{\sigma+z} \cdot \frac{1}{2} \frac{g\left(g \bar{p}+\sqrt{g^{2} \bar{p}^{2}-8-4 z}\right)}{z} .
$$

Then using (43), (33) and (28), the Stieltjes transform of the asymptotic distribution of eigenvalues of $\hat{\mathbf{R}} \mathbf{P}^{1 / 2} \hat{\mathbf{R}}_{1} \mathbf{P}^{1 / 2}$ in (19) is obtained. Using free probability theory, the $\mathbf{R}$ transform of the density of the eigenvalues of $\hat{\mathbf{R}}_{\mathbf{Y}}$, given by (14), can be written as [46]

$$
\mathcal{R}_{\hat{\mathbf{R}}_{\mathbf{Y}}}(z)=\mathcal{R}_{\mathbf{W}}(z)+\mathcal{R}_{\hat{\mathbf{R}}_{\mathbf{Z}}}(z)
$$

Finally, the expression for the Stieltjes transform in (20) is obtained using the definitions of transforms in the Appendix A and (19).

\section{APPENDIX D}

\section{Proof of Theorem 5.1}

We use $\mathrm{R}$ transform and $\Sigma$ transform to derive the aepdf of $\hat{\mathbf{R}}_{\mathbf{Y}}$ using free probability theory. Since $\hat{\mathbf{R}}$ and $\hat{\mathbf{R}}_{1}$ follow the MP law, their $\Sigma$ transforms can be calculated using (12). The $\Sigma$ transform of $\Theta$, which follows the tilted semicircular law (22) is given by [51];

$$
\Sigma_{\Theta}(z)=1-\mu z
$$

It can be noted that since $\Theta$ and $\hat{\mathbf{R}}_{1}$ are square matrices, the terms $\Theta^{1 / 2} \hat{\mathbf{R}}_{1} \Theta^{1 / 2}$ and $\Theta \hat{\mathbf{R}}_{1}$ have identical eigenvalues [46]. Then the $\Sigma$ transform of the product of $\hat{\mathbf{R}}, \Theta$ and $\hat{\mathbf{R}}_{1}$ can be written as

$$
\Sigma_{\hat{\mathbf{R}} \Theta \hat{\mathbf{R}}_{1}}(z)=\frac{1}{\rho+z} \cdot(1-\mu z) \cdot \frac{1}{\sigma+z} .
$$

Subsequently, the polymonial for the corresponding $\eta$ transform is obtained using (28) and (46), which is given by

$$
\eta(\rho+\eta-1)(\eta+\sigma-1) z+(\eta-1)(1-\mu(\eta-1))=0
$$

The Stieltjes transform of the density of the eigenvalues of the product of $\hat{\mathbf{R}}$ and $\Theta \hat{\mathbf{R}}_{1}$ can be obtained using (47) and (33) and is given by

$$
\begin{array}{r}
z^{2} \mathcal{S}^{3}(z)+\left(-\mu z^{2}-\sigma z+2 z-\rho z\right) \mathcal{S}^{2} \\
+(-\rho+1+\rho \sigma-2 \mu z-\sigma-z) \mathcal{S}(z)-1-\mu .
\end{array}
$$


The $\mathrm{R}$ transform of the density of the eigenvalues of the product of $\hat{\mathbf{R}}$ and $\boldsymbol{\Theta} \hat{\mathbf{R}}_{1}$ is calculated using (29) and (48) and is given by

$$
\mathcal{R}_{\hat{\mathbf{R}} \Theta \hat{\mathbf{R}}_{1}}(z)=-\frac{1}{2} \frac{\left(-1+z \sigma+z \rho+\sqrt{1-2 z \sigma-2 z \rho+z^{2} \sigma^{2}-2 z^{2} \rho \sigma+z^{2} \rho^{2}-4 \rho \sigma z \mu}\right)}{(z(z+\mu))} .
$$

Since the term $\hat{\mathbf{R}}_{\mathbf{Z}}$ in (23) follows the MP distribution, the $\mathrm{R}$ transform of $\hat{\mathbf{R}}_{\mathbf{Y}}$ using additive free convolution property can be written as

$$
\mathcal{R}_{\hat{\mathbf{R}}_{\mathbf{Y}}}(z)=p \mathcal{R}_{\hat{\mathbf{R}}_{\Theta} \hat{\mathbf{R}}_{1}}(p z)+\mathcal{R}_{\hat{\mathbf{R}}_{\mathbf{Z}}}(z) .
$$

Finally, the Stieltjes transform of the density of $\hat{\mathbf{R}}_{\mathbf{Y}}$ can be calculated using (50) and (29).

\section{REFERENCES}

[1] A. Goldsmith, S. Jafar, I. Maric, and S. Srinivasa, "Breaking spectrum gridlock with cognitive radios: An information theoretic perspective," Proc. IEEE, vol. 97, no. 5, pp. 894 -914, May 2009.

[2] T. Yucek and H. Arslan, "A survey of spectrum sensing algorithms for cognitive radio applications," IEEE Commun. Surveys Tutorials, vol. 11, no. 1, pp. $116-130$, quarter 2009.

[3] Y. Zeng and Y. chang Liang, "Eigenvalue-based spectrum sensing algorithms for cognitive radio," IEEE Trans. Commun., vol. 57, no. 6, pp. $1784-1793$, June 2009.

[4] S. K. Sharma, S. Chatzinotas, and B. Ottersten, "Exploiting polarization for spectrum sensing in cognitive SatComs," in 7th Int. Conf. CROWNCOM, June 2012, pp. $36-41$.

[5] S. K. Sharma, S. Chatzinotas, and B. Ottersten, "Spectrum sensing in dual polarized fading channels for cognitive SatComs," in IEEE Globecom Conf., Dec. 2012, pp. 3419 -3424.

[6] D. Cabric and R. Brodersen, "Physical layer design issues unique to cognitive radio systems," in IEEE 16th Int. Symp. Personal, Indoor and Mobile Radio Commun., Sept. 2005, pp. $759-763$.

[7] H. Tang, "Some physical layer issues of wide-band cognitive radio systems," in First IEEE Int. Symp. New Frontiers in Dynamic Spectrum Access Networks, Nov. 2005, pp. $151-159$.

[8] R. Walden, "Analog-to-digital converter survey and analysis," IEEE Journal on Selected Areas in Communications, vol. 17, no. 4, pp. 539 -550, April 1999.

[9] M. Ariaudo, I. Fijalkow, J. Gautier, M. Brandon, B. Aziz, and B. Milevsky, "Green radio despite Dirty RF front-end, EURASIP J. Wireless Commun. and Networking, vol. 146, 2012.

[10] Z. Tian and G. Giannakis, "Compressed sensing for wideband cognitive radios," in IEEE Int. Conf. Acoustics, Speech and Signal Process., vol. 4, April 2007, pp. $1357-1360$.

[11] H. Sun, A. Nallanathan, C. Wang and Y. Chen, "Wideband spectrum sensing for cognitive radio networks: a survey," IEEE Wireless Communications, vol. 20, no. 2, pp.74-81, April 2013.

[12] D. Donoho, "Compressed sensing," IEEE Trans. Info. Th., vol. 52, no. 4, pp. 1289 -1306, April 2006.

[13] E. J. Cands and M. Wakin, "An introduction to compressive sampling," IEEE Signal Process. Magazine, vol. 25, no. 2, pp. 21-30, March 2008.

[14] S. K. Sharma, M. Patwary and M. Abdel-Maguid, "Spectral efficient compressive transmission framework for wireless communication systems", IET Signal Process., vol. 7, no. 7. pp. 558-564, Sept. 2013.

[15] J. Tropp and A. Gilbert, "Signal recovery from random measurements via orthogonal matching pursuit," IEEE Trans. Info. Th., vol. 53, no. 12, pp. 4655 -4666, Dec. 2007.

[16] W. Dai and O. Milenkovic, "Subspace pursuit for compressive sensing signal reconstruction," IEEE Trans. Info. Th., vol. 55, no. 5, pp. 2230 -2249, May 2009.

[17] Z. Zeinalkhani and A. Banihashemi, "Iterative recovery algorithms for compressed sensing of wideband block sparse spectrums," in IEEE Int. Conf. Commun. (ICC), June 2012, pp. $1630-1634$.

[18] K. Patil, R. Prasad, and K. Skouby, "A survey of worldwide spectrum occupancy measurement campaigns for cognitive radio," in Int. Conf. Devices and Commun. (ICDeCom), Feb. 2011, pp. 1 -5.

[19] Y. Wang, A. Pandharipande, Y. Polo, and G. Leus, "Distributed compressive wide-band spectrum sensing," in Info. Theory and Applications Workshop, Feb., 2009, pp. 178-183.

[20] M. Davenport, P. Boufounos, M. Wakin, and R. Baraniuk, "Signal processing with compressive measurements," IEEE J. Sel. Topics Signal Process., vol. 4, no. 2, pp. 445-460, Feb. 2010.

[21] J. E. Vila-Forcen, A. Artes-Rodriguez, and J. Garcia-Frias, "Compressive sensing detection of stochastic signals," in 42nd Annual Conf. Info. Sciences and Systems, March 2008, pp. 956-960.

[22] S. Hong, "Direct spectrum sensing from compressed measurements," in Military Commun. Conf., Oct.- Nov. 2010 , pp. 1187-1192.

[23] Y. Wang, Z. Tian, and C. Feng, "Sparsity order estimation and its application in compressive spectrum sensing for cognitive radios," IEEE Trans. Wireless Commun., vol. 11, no. 6, pp. 2116 -2125, June 2012. 
[24] S. K. Sharma, S. Chatzinotas, and B. Ottersten, "Eigenvalue based sensing and SNR estimation for cognitive radio in presence of noise correlation," IEEE Trans. Veh. Technol., vol. 62, no. 8, pp. 3671-3684, Oct. 2013.

[25] A. Tulino, G. Caire, S. Verdu, and S. Shamai, "Support recovery with sparsely sampled free random matrices," IEEE Trans. Info. Th., vol. 59, no. 7, pp. 4243-4271, July 2013.

[26] Y. Eldar, "Compressed sensing of analog signals in shift-invariant spaces," IEEE Trans. Signal Process., vol. 57, no. 8, pp. 2986 -2997, Aug. 2009.

[27] H. Sun, W.-Y. Chiu, J. Jiang, A. Nallanathan, and H. Poor, "Wideband spectrum sensing with sub-Nyquist sampling in cognitive radios," IEEE Trans. Signal Process., vol. 60, no. 11, pp. 6068 -6073, Nov. 2012.

[28] M. Mishali and Y. Eldar, "From theory to practice: Sub-Nyquist sampling of sparse wideband analog signals," IEEE J. Sel. Topics Signal Process., vol. 4, no. 2, pp. 375 -391, April 2010.

[29] D. Malioutov, S. Sanghavi, and A. Willsky, "Compressed sensing with sequential observations," in IEEE Int. Conf. Acoustics, Speech and Signal Process., 2008, pp. $3357-3360$.

[30] D. Malioutov, M. Cetin, and A. Willsky, "A sparse signal reconstruction perspective for source localization with sensor arrays," IEEE Trans. Signal Process., vol. 53, no. 8, pp. 3010-3022, 2005.

[31] C. Qi, X. Wang, and L. Wu, "Underwater acoustic channel estimation based on sparse recovery algorithms," IET Signal Process., vol. 5, no. 8, pp. 739-747, 2011.

[32] S. Wright, R. Nowak, and M. Figueiredo, "Sparse reconstruction by separable approximation," IEEE Trans. Signal Process., vol. 57, no. 7, pp. 2479-2493, 2009 .

[33] S.-J. Kim, K. Koh, M. Lustig, S. Boyd, and D. Gorinevsky, "An interior-point method for large-scale 11-regularized least squares," IEEE J. Sel. Topics Signal Process., vol. 1, no. 4, pp. 606-617, 2007.

[34] Z. He, A. Cichocki, R. Zdunek, and S. Xie, "Improved focuss method with conjugate gradient iterations," IEEE Trans. Signal Process., vol. 57, no. 1, pp. 399-404, 2009

[35] H. Zhu, G. Leus, and G. Giannakis, "Sparsity-cognizant total least-squares for perturbed compressive sampling," IEEE Trans. Signal Process., vol. 59, no. 5, pp. 2002-2016, 2011.

[36] S. Cotter, B. Rao, K. Engan, and K. Kreutz-Delgado, "Sparse solutions to linear inverse problems with multiple measurement vectors," IEEE Trans. Signal Process., vol. 53, no. 7, pp. 2477-2488, 2005.

[37] Y. Eldar and M. Mishali, "Robust recovery of signals from a structured union of subspaces," IEEE Trans. Info. Th., vol. 55, no. 11, pp. $5302-5316$, Nov. 2009.

[38] D. Wipf and B. Rao, "An empirical bayesian strategy for solving the simultaneous sparse approximation problem," IEEE Trans. Signal Process., vol. 55, no. 7, pp. 3704-3716, 2007.

[39] J. Romberg, "Compressive sensing by random convolution," SIAM J. Imag. Sci., vol. 2, no. 4, pp. 1098-1128, 2009.

[40] J. Tropp, J. Laska, M. Duarte, J. Romberg, and R. Baraniuk, "Beyond Nyquist: Efficient sampling of sparse bandlimited signals," IEEE Trans. Info. Th., vol. 56, no. 1, pp. 520 -544, Jan. 2010.

[41] G. Reeves and M. Gastpar, "The sampling rate-distortion tradeoff for sparsity pattern recovery in compressed sensing," IEEE Trans. Info. Th., vol. 58, no. 5, pp. 3065-3092, 2012.

[42] J. Yuzhe, and B. D. Rao, "Support Recovery of Sparse Signals in the Presence of Multiple Measurement Vectors," IEEE Trans. Info. Th., vol. 59, no. 5, pp. 3139-3157, May 2013.

[43] A. Fletcher, S. Rangan, and V. Goyal, "Necessary and sufficient conditions for sparsity pattern recovery," IEEE Trans. Info. Th., vol. 55, no. 12 , pp. 5758-5772, 2009.

[44] W. Wang, M. Wainwright, and K. Ramchandran, "Information-theoretic limits on sparse signal recovery: Dense versus sparse measurement matrices," IEEE Trans. Info. Th., vol. 56, no. 6, pp. 2967-2979, 2010.

[45] X. Yu and S. J. Baek, "Sufficient conditions on stable recovery of sparse signals with partial support information," IEEE Signal Process. Letters, vol. 20, no. 5, pp. 539-542, 2013.

[46] A. M. Tulino and S. Verdu, "Random matrix theory and wireless communications," Foundations and Trends in Commun. and Info. Th., vol. 1, no. 1, pp. 1-182, 2004.

[47] J. Meng, W. Yin, H. Li, E. Hossain, and Z. Han, "Collaborative spectrum sensing from sparse observations in cognitive radio networks," IEEE J. Sel. Areas Commun., vol. 29, no. 2, pp. 327 -337, Feb. 2011.

[48] S. K. Sharma, S. Chatzinotas, and B. Ottersten, "SNR Estimation for Multi-dimensional Cognitive Receiver under Correlated Channel/Noise", IEEE Trans. Wilress Commun., vol. 12, no. 12, pp. 6392-6405, Dec. 2013.

[49] S. K. Sharma, S. Chatzinotas, and B. Ottersten, "Eigenvalue based SNR estimation for cognitive radio in presence of channel correlation," in IEEE Global Commun. Conf., 2013, pp. 1129-1134.

[50] S. Chatzinotas, M. Imran, and R. Hoshyar, "On the multicell processing capacity of the cellular MIMO uplink channel in correlated Rayleigh fading environment," IEEE Trans. Wireless Commun., vol. 8, no. 7, pp. 3704 -3715, July 2009.

[51] X. Mestre, J. Fonollosa, and A. Pages-Zamora, "Capacity of MIMO channels: asymptotic evaluation under correlated fading," IEEE J. Sel. Areas Commun., vol. 21, no. 5, pp. 829 - 838, June 2003

[52] R. Couillet and M. Debbah, "Random Matrix Methods for Wireless Communications", Cambridge University Press, 2011.

[53] D. Voiculescu, "Multiplication of certain non-commuting random variables," J. Operator Theory, vol. 18, pp. 223-235, 1987.

[54] D. Voiculescu, "Addition of certain non-commuting random variables," J. Funct. Analysis, vol. 66, pp. 323-346, 1986.

[55] S. K. Sharma, S. Chatzinotas, and B. Ottersten, "The effect of noise correlation on fractional sampling based spectrum sensing," in IEEE Int. Conf. Commun., June 2013, pp. 1182-1187.

[56] Z. Zhang and B. Rao, "Sparse signal recovery in the presence of correlated multiple measurement vectors," in IEEE Int. Conf. Acoustics Speech and Signal Process., March 2010, pp. 3986-3989. 


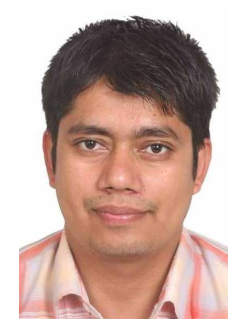

Shree Krishna Sharma (S'12) received the B.E. degree in electronics and communication from Birla Institute of Technology, Mesra, India, in 2004; the M.Sc. degree in information and communication engineering from the Institute of Engineering, Pulchowk, Nepal, in 2010; the M.A. degree in economics from Tribhuvan University Patan Multiple Campus, Patan, Nepal, and the M.Res. degree in computing science from Staffordshire University, Staffordshire, U.K., in 2011. He is currently working toward the Ph.D. degree with the Interdisciplinary Centre for Security, Reliability and Trust, University of Luxembourg, Luxembourg City, Luxembourg.

In the past, he was also involved with Kathmandu University, Dhulikhel, Nepal, as a Teaching Assistant, and he served as a Part-Time Lecturer for eight engineering colleges in Nepal. He was with Nepal Telecom for more than four years as a Telecom Engineer in the field of information technology and telecommunication. His research interests include cognitive wireless communications, resource allocation, and interference mitigation in heterogeneous wireless networks. Mr. Sharma received an Indian Embassy Scholarship for his B.E. study, an Erasmus Mundus Scholarship for his M.Res. study, and an Aids TrainingResearch Ph.D. grant from the National Research Fund of Luxembourg.

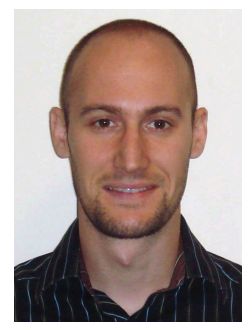

Symeon Chatzinotas (S'06-M'09-SM'13) Symeon Chatzinotas (S06-M09-SM13) received the M.Eng. degree in telecommunications from Aristotle University of Thessaloniki, Thessaloniki, Greece, in 2003 and the M.Sc. and the $\mathrm{Ph.D}$. degrees in electronic engineering from the University of Surrey, Surrey, U.K., in 2009. He is currently a Research Scientist with the Interdisciplinary Centre for Security, Reliability and Trust, University of Luxembourg.

In the past, he has worked on numerous research and development projects for the Institute of Informatics and Telecommunications, National Center for Scientific Research Demokritos, Athens, Greece; the Institute of Telematics and Informatics, Center of Research and Technology Hellas, Thessaloniki, Greece; and the Mobile Communications Research Group, Center of Communication Systems Research, University of Surrey. He is the author of more than 110 technical papers in refereed international journals, conferences, and scientific books and he is currently coediting a book on "Cooperative and Cognitive Satellite Systems". His research interests include multiuser information theory, cooperative and cognitive communications, and transceiver optimization for terrestrial and satellite networks.

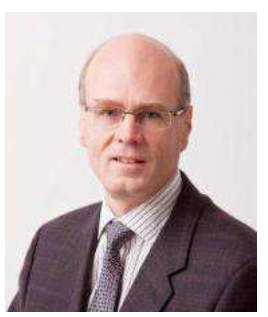

Björn Ottesten (S'87-M'89-SM'99-F'04) was born in Stockholm, Sweden, in 1961. He received the M.S. degree in electrical engineering and applied physics from Linköping University, Linköping, Sweden, in 1986 and the Ph.D. degree in electrical engineering from Stanford University, Stanford, CA, in 1989.

Dr. Ottersten has held research positions at the Department of Electrical Engineering, Linköping University, the Information Systems Laboratory, Stanford University, the Katholieke Universiteit Leuven, Leuven, and the University of Luxembourg. During 96/97, he was Director of Research at ArrayComm Inc, a start-up in San Jose, California based on Ottersten's patented technology. He has co-authored journal papers that received the IEEE Signal Processing Society Best Paper Award in 1993, 2001, 2006, and 2013 and 3 IEEE conference papers receiving Best Paper Awards. In 1991, he was appointed Professor of Signal Processing at the Royal Institute of Technology (KTH), Stockholm. From 1992 to 2004, he was head of the department for Signals, Sensors, and Systems at KTH and from 2004 to 2008 , he was dean of the School of Electrical Engineering at KTH. Currently, he is Director for the Interdisciplinary Centre for Security, Reliability and Trust at the University of Luxembourg. As Digital Champion of Luxembourg, he acts as an adviser to European Commissioner Neelie Kroes.

Dr. Ottersten has served as Associate Editor for the IEEE TRANSACTIONS ON SIGNAL PROCESSING and on the editorial board of IEEE Signal Processing Magazine. He is currently editor in chief of EURASIP Signal Processing Journal and a member of the editorial boards of EURASIP Journal of Applied Signal Processing and Foundations and Trends in Signal Processing. He is a Fellow of the IEEE and EURASIP and a member of the IEEE Signal Processing Society Board of Governors. In 2011, he received the IEEE Signal Processing Society Technical Achievement Award. He is a first recipient of the European Research Council advanced research grant. His research interests include security and trust, reliable wireless communications, and statistical signal processing. 